\title{
Temporal Groupwise Registration for Motion Modeling
}

\author{
Mehmet Yigitsoy ${ }^{\star}$, Christian Wachinger ${ }^{\star}$, and Nassir Navab \\ Computer Aided Medical Procedures (CAMP), Technische Universität München \\ \{yigitsoy, wachinger, navab\}@in.tum.de
}

\begin{abstract}
We propose a novel method for the registration of time-resolved image sequences, called Spatio-Temporal grOupwise non-rigid Registration using freeform deforMations (STORM). It is a groupwise registration method, with a group of images being considered simultaneously, in order to prevent bias introduction. This is different from pairwise registration methods where only two images are registered to each other. Furthermore, STORM is a spatio-temporal registration method, where both, the spatial and the temporal information are utilized during the registration. This ensures the smoothness and consistency of the resulting deformation fields, which is especially important for motion modeling on medical data. Moreover, popular free-form deformations are applied to model the nonrigid motion. Experiments are conducted on both synthetic and medical images. Results show the good performance and the robustness of the proposed approach with respect to outliers and imaging artifacts, and moreover, its ability to correct for larger deformation in comparison to standard pairwise techniques.
\end{abstract}

\section{Introduction}

In radiation therapy, target localization is one of the biggest challenges, since the exposition of healthy tissue to ionizing radiation should be kept as low as possible [5]. Organ motion due to respiration, however, can lead to inaccuracies during target localization [8]. Modeling and analyzing organ motion is therefore important for such applications. Thanks to dynamic imaging, which provides time-resolved images of the anatomy, the modeling of organ motion becomes feasible. Time-resolved images are extensively used to study cardiac [10,11, 16], lung [3, 4, 8] and liver [17, 26] motion.

The creation of motion models necessitates the non-rigid alignment of time-resolved volumes. The standard approach is, first, to perform pairwise registrations, and second, to combine the deformation fields to create the motion model. This can result in inconsistent and non-smooth motion fields along the temporal direction [22]. In applications, such as radiotherapy, it is, however, crucial to ensure smoothness and consistency over time to estimate the delivered dose accurately at any time point in the image sequence [14]. Further, the pairwise registration approach has the disadvantage that either, all registrations are performed towards one target leading to large displacements fields, or, registrations between adjacent volumes are calculated causing the accumulation of errors in the image sequence [4].

Methods that address the issue of incorporating temporal information [1] 13] still have the problem of fixed reference image selection. Although there are spatio-temporal

\footnotetext{
* Joint first authors.

G. Székely and H.K. Hahn (Eds.): IPMI 2011, LNCS 6801, pp. 648-659 2011.

(c) Springer-Verlag Berlin Heidelberg 2011
} 
methods that avoid the selection of a reference image in the sequence, either their main focus is to register two different image sequences [15, 16], or to register two image sequences with the second being the replication of the reference image [22]. On the other hand, groupwise registration techniques are proposed for atlas construction that address the choice of the reference image [9]230]. Although, their application areas are quite different from motion modeling, the main goal of these approaches is to reduce the bias introduced by a fixed reference image [9,6].

In this paper, we propose a new registration method for the deformable alignment of time-resolved images. Instead of applying pairwise registrations, we perform a fully simultaneous, groupwise registration of all images in the sequence. This eliminates the bias introduced by reference image selection and the error accumulation during the sequential registration. The second novelty of our approach addresses the need for a smooth deformation field. Instead of having a separate deformation field for each image, we create one deformation field that is one dimension higher than the image dimension. Since we work with free-form-deformations (FFD) based on B-splines, this intrinsically ensures that we obtain a smooth deformation field at each update step, also along the temporal direction. This leads to further advantages for the registration method because it is more robust to outliers, can handle large deformations, and allows for temporal interpolation, which differentiates it from existing approaches.

\section{Related Work}

Approaches aiming at the registration of time-resolved image sequences mainly focus on $4 \mathrm{D}$ modeling of organ motion of heart, liver or lung. Klein et al. [11] use 4D deformable image registration to compensate for the cardiac motion in positron emission tomography (PET) images, following a pairwise approach. Rohlfing et al. [17] apply intensity-based deformable registration on gated magnetic resonance (MR) images to model liver motion during a respiratory cycle. They register each volumetric phase image to a reference image by using the displacement field computed for the previous volumetric image as the initial deformation. Sarrut et al. [20] simulate a 4D computed tomography (CT) image of the thorax by first registering end-diastole and end-systole images and then by linearly interpolating images in between. These methods either do not use temporal information or fix a reference frame during the registration. Sundar et al. [22] addresses the temporal smoothness problem by considering motion estimation from cine MRI cardiac images as the registration of two 3D image sequences. The second sequence was, however, created by replicating the end-diastolic image. This means that although this method addresses the problem of temporal smoothness, the existence of the reference image still remains an issue. Wu et al. [29] propose a groupwise registration method for longitudinal image sequences where a smoothing kernel is used to assure temporal consistency.

Simultaneous registration approaches align groups of images without the necessity of reference selection. Learned-Miller [12] proposes the congealing framework for image registration, which sums up the entropy of pixel stacks over the image. Zöllei et al. [30] apply this method to the affine registration of population data. Balci et al. [2] extend this method by including free-form deformations to the deformable registration 
of a group of brain images. Wachinger et al. [27] perform simultaneous registration by accumulating pairwise estimates for the similarity estimation. All these methods for simultaneous registration are not designed for motion estimation, and consequently, do not take temporal information into account. Bystrov et al. [3] try to address both aspects, temporal smoothness and reference image selection, but the method is limited to local motion trajectories.

\section{Method}

Assuming $n$ images $I_{1}, \ldots, I_{n}$, with $I_{i}: \Omega \rightarrow \mathbb{R}, \Omega \subset \mathbb{R}^{N}$, we stack the images and create an image $I$ of dimension $N+1, I=\left[I_{1}, I_{2}, \ldots, I_{n}\right]$,with $I: \Omega \times\{1, \ldots, n\} \rightarrow \mathbb{R}$. The transformation $\mathbf{T}: \mathbb{R}^{N+1} \rightarrow \mathbb{R}^{N+1}$ is defined as

$$
\mathbf{T}_{\Phi}(\mathbf{x})=\mathbf{x}+\mathbf{D}_{\Phi}(\mathbf{x})
$$

with the spatial coordinate $\mathbf{x}=\left[x_{1}, x_{2}, \cdots, x_{N+1}\right] \in I$ and $\mathbf{D}_{\Phi}$ the deformation field with deformation parameters $\Phi$. We denote the warped images with $I^{\downarrow}(\mathbf{x})=I(\mathbf{T}(\mathbf{x}))$. In the following sections, we describe the main components of our registration method: transformation model, similarity metric, and optimization method.

\subsection{Transformation Model}

We use FFD B-Splines to model non-rigid motion [19], with an additional dimension along the temporal direction. For the temporal direction, we can set B-Spline functions of a different order or we can adapt the spacing between the control points to model the desired smoothness along the temporal direction. $\Phi$ is defined as a grid of $n_{1} \times n_{2} \times$ $\cdots \times n_{N+1}$ control points on image $I$ with spacings $\delta_{1}, \delta_{2}, \cdots, \delta_{N+1}$. We access a control point with $\mathbf{i}=\left[i_{1}, i_{2}, \cdots, i_{N+1}\right]$. Further, using cubic B-splines we obtain

$$
D_{\Phi}(\mathbf{x})=\sum_{a_{1}=0}^{3} \sum_{a_{2}=0}^{3} \ldots \sum_{a_{N+1}=0}^{3} B_{a_{1}}\left(r_{1}\right) B_{a_{2}}\left(r_{2}\right) \cdots B_{a_{N+1}}\left(r_{N+1}\right) \cdot \Phi(\mathbf{i}+\mathbf{a})
$$

where

$$
\begin{gathered}
i_{1}=\left\lfloor\frac{x_{1}}{\delta_{1}}\right\rfloor-1, i_{2}=\left\lfloor\frac{x_{2}}{\delta_{2}}\right\rfloor-1, \cdots, i_{N+1}=\left\lfloor\frac{x_{N+1}}{\delta_{N+1}}\right\rfloor-1, \\
r_{1}=\frac{x_{1}}{\delta_{1}}-\left\lfloor\frac{x_{1}}{\delta_{1}}\right\rfloor, r_{2}=\frac{x_{2}}{\delta_{2}}-\left\lfloor\frac{x_{2}}{\delta_{2}}\right\rfloor, \cdots, r_{N+1}=\frac{x_{N+1}}{\delta_{N+1}}-\left\lfloor\frac{x_{N+1}}{\delta_{N+1}}\right\rfloor,
\end{gathered}
$$

$B_{0}$ to $B_{3}$ are cubic $\mathrm{B}$-spline basis functions, and $\mathbf{a}=\left[a_{1}, a_{2}, \cdots, a_{N+1}\right]$. For the interpolation of $N+1$ dimensional data, we have $\Phi(\mathbf{i})=\left(\phi_{x_{1}}^{\mathbf{i}}, \phi_{x_{2}}^{\mathbf{i}}, \cdots, \phi_{x_{N+1}}^{\mathbf{i}}\right)^{\top}$.

\subsection{Accumulated Pairwise Estimates}

We apply accumulated pairwise estimates (APE) [27] as similarity measure. It was introduced as a framework for groupwise similarity metrics. In order to describe it in the scope of this temporally extended framework, we use the following notation. As before, 
we consider $\mathbf{x}$ to be a point in $\Omega \times\{1, \ldots, n\}$. Further, be $\mathbf{v} \in \Omega$ and $g, h \in\{1, \ldots, n\}$. The point $\mathbf{x}$ that is related to the spatial location $\mathbf{v}$ and the temporal location $g$ is denoted by $\mathbf{x}_{\mathbf{v}}^{g}=[\mathbf{v}, g]$. Therefore, we can access the same pixel by $I_{g}(\mathbf{v})=I\left(\mathbf{x}_{\mathbf{v}}^{g}\right)$, leading to the cost function

$$
\begin{aligned}
\mathcal{C}(\Phi) & =\sum_{g=1}^{n} \sum_{\substack{h=1 \\
h \neq g}}^{n}\left\|I_{g}^{\downarrow}-I_{h}^{\downarrow}\right\|^{2} \\
& =\sum_{g=1}^{n} \sum_{\substack{h=1 \\
h \neq g}}^{n} \sum_{\mathbf{v} \in \Omega}\left(I\left(\mathbf{T}_{\Phi}\left(\mathbf{x}_{\mathbf{v}}^{g}\right)\right)-I\left(\mathbf{T}_{\boldsymbol{\Phi}}\left(\mathbf{x}_{\mathbf{v}}^{h}\right)\right)\right)^{2} .
\end{aligned}
$$

\subsection{Optimization}

Simultaneous registration leads to a computationally challenging optimization problem because of the increase of the parameter space and the more complex evaluation of the similarity metric. For this reason, we use a gradient-based optimization procedure in combination with a stochastic sampling in the spatial domain [25, 30, 2]. The stochastic sampling significantly reduces the computational cost, where we randomly select $10 \%$ of the total pixels in $\Omega$ in each iteration on which the similarity is calcualted. It is further noted in [25] that the noisy estimate of the gradient can reduce the problem of local minima. The update equation is

$$
\Phi \leftarrow \Phi+\tau \cdot \nabla \mathcal{C}
$$

with the step length $\tau$, which is determined by a line search following the Wolfe conditions. The partial derivative with respect to control point $\phi^{\mathbf{i}}$ along direction $x_{1}$, with other directions analogously, is

$$
\begin{gathered}
\frac{\partial \mathcal{C}(\Phi)}{\partial \phi_{x_{1}}^{\mathbf{i}}}=\frac{\partial}{\partial \phi_{x_{1}}^{\mathbf{i}}} \sum_{g=1}^{n} \sum_{\substack{h=1 \\
h \neq g}}^{n} \sum_{\mathbf{v} \in \Omega}\left(I^{\downarrow}\left(\mathbf{x}_{\mathbf{v}}^{g}\right)-I^{\downarrow}\left(\mathbf{x}_{\mathbf{v}}^{h}\right)\right)^{2} \\
\frac{\partial\left(I^{\downarrow}\left(\mathbf{x}_{\mathbf{v}}^{g}\right)-I^{\downarrow}\left(\mathbf{x}_{\mathbf{v}}^{h}\right)\right)^{2}}{\partial \phi_{x_{1}}^{\mathbf{i}}}=2\left(I^{\downarrow}\left(\mathbf{x}_{\mathbf{v}}^{g}\right)-I^{\downarrow}\left(\mathbf{x}_{\mathbf{v}}^{h}\right)\right) \frac{\partial\left(I^{\downarrow}\left(\mathbf{x}_{\mathbf{v}}^{g}\right)-I^{\downarrow}\left(\mathbf{x}_{\mathbf{v}}^{h}\right)\right)}{\partial \phi_{x_{1}}^{\mathbf{i}}} \\
\frac{\partial\left(I^{\downarrow}\left(\mathbf{x}_{\mathbf{v}}^{g}\right)-I^{\downarrow}\left(\mathbf{x}_{\mathbf{v}}^{h}\right)\right)}{\partial \phi_{x_{1}}^{\mathbf{i}}}=\left(\nabla_{x_{1}} I^{\downarrow}\left(\mathbf{x}_{\mathbf{v}}^{g}\right) \frac{\partial \mathbf{T}_{\Phi}\left(\mathbf{x}_{\mathbf{v}}^{g}\right)}{\partial \phi_{x_{1}}^{\mathbf{i}}}-\nabla_{x_{1}} I^{\downarrow}\left(\mathbf{x}_{\mathbf{v}}^{h}\right) \frac{\partial \mathbf{T}_{\Phi}\left(\mathbf{x}_{\mathbf{v}}^{h}\right)}{\partial \phi_{x_{1}}^{\mathbf{i}}}\right) \\
\frac{\partial \mathbf{T}_{\Phi}\left(\mathbf{x}_{\mathbf{v}}^{g}\right)}{\partial \phi_{x_{1}}^{\mathbf{i}}}
\end{gathered}
$$

with $r_{1}^{g}, r_{2}^{g}, \cdots, r_{N+1}^{g}$ as defined in equation (4) with respect to the location $\mathbf{x}_{\mathbf{v}}^{g}$. We position the images along the temporal direction, reflecting its acquisition time. Alternatively, one could decide to use different criteria to position the images along the temporal direction, such as amount of change. For some applications it may further be useful to allow for deformations along the temporal direction, if there is uncertainty about the exact acquisition time, or different parts of the image were acquired at different time points. For our application, we do not need this flexibility so that we set the update along the temporal direction to zero. 


\subsection{Implementation Details}

We apply a multi-resolution approach to reduce computational cost and increase robustness to local minima. Further, we apply a multi-grid approach, with several grid refinements on each image resolution, to capture large displacements, as well as, fine details. In order to guarantee smoothness and consistency over time, we use a a single control point grid of one higher dimension and overlay this onto the $(N+1) \mathrm{D}$ image. For the temporal dimension, we use fewer control points than the number of images to increase the dependency between the images and, at the same time, to further decrease the computational cost. The software is developed using open-source libraries ITK and VTK. The application will be made publicly available in the near future.

\subsection{Advantages of STORM for Motion Modeling}

After having described the method, we want to point out the advantages of STORM for motion modeling.

1. Smooth and Temporally Consistent Deformation Field: Current approaches perform first the registration of the images, and then the combination and regularization of the transformation fields. The problem is that for the regularization step, the image information is no longer considered, and therefore transformation fields may be created that do not match to the underlying images. In contrast, our single Bspline deformation field across all the images intrinsically guarantees a smooth and consistent transformation along the temporal direction in each update step. Similarity and regularization aspects are jointly optimized.

2. Robustness Against Outliers: Dynamic imaging is more challenging than its static counterpart, causing more artifacts and noise in the images. Our method is robust to such effects because the $(N+1) \mathrm{D}$ deformation field interlaces neighboring images, with the number of control points $n_{N+1}$ determining to which extent. Images containing outliers can therefore still be correctly aligned, if neighboring images drive the registration.

3. Handling Large Displacements: During the registration of time-resolved images, one of the main issues is the registration of distant images. If the displacement between the images is too large, pairwise methods fail to find the mapping. By incorporation temporal information and using groupwise registration, STORM is able to better handle large displacements in the sequence.

4. Time Interpolation: In some applications, it is desirable to see the organ at a specific state of the motion [7, 21]. However, due to the low temporal resolution of time-resolved images, this is not always possible. Thanks to the higher dimensional deformation field of STORM, it is feasible to resample images for any time point by applying the inverse mapping.

5. Tracking Using Deformation Field: In 4D radiation therapy, it is necessary to extract motion trajectories of a tumor region, in order to perform the pre-operative dosimetry planning [8]. Our approach enables tracking of certain structures by clipping the $(N+1)$ D deformation field along the temporal dimension. 


\section{Experiments and Results}

In order to evaluate the performance of STORM, we experiment with temporally-related synthetic and medical data sets. In addition to using visual inspection for qualitative evaluation, mean and standard deviation (STD) along the temporal direction are computed for the quantitative validation. Due to space limitations, every second image is shown for each sequence.

\subsection{Synthetic Data}

Synthetic image sequences are created by applying gradually increasing deformations to a template image to simulate a smooth deformation over time, reflecting a smooth motion. The experiments are conducted to demonstrate the advantages listed in section 3.5 For each experiment a multi-resolution setting with 30 iterations on the highest level that is increased by a factor of 1.2 for each lower level is used together with 8 control points in spatial dimensions and 7 control points in the temporal dimension.

\section{Growing Ring}

The input images shown in Fig. 1 are binary images of a continuously growing ring of size $100 \times 100$ pixels. The registered images are also shown in the same figure. We observe that all images are correctly aligned to the initially unknown target. The wireframe meshes are presented to visualize the deformations. The direction of the displacements is encoded in the meshes with the hue component. Mean and STD images, calculated along the temporal direction, confirm the correct registration. The mean image clearly shows the ring, and the STD image only shows small deviations on the edges of the ring caused by interpolation artifacts. The $(N+1) \mathrm{D}$ image and the control point grid are shown in Figs. 2(a) and 2(b), respectively. The visualization of the ND meshes in $(N+1) \mathrm{D}$ is shown in Fig. 2(c) The deformation along the temporal direction can be observed by clipping the $(N+1) \mathrm{D}$ deformed mesh along the time-axis as shown in Fig. 2(d) The smoothness and consistency of the deformation along the temporal dimension in this figure, as well as, the smoothness of the change of colors in Fig. 2(c) demonstrates the advantages of our methods described in 3.51 and 3.55 .

\section{Black Sheep}

In this experiment, the same growing ring sequence is used with an outlier image introduced to create a 'black sheep' in the sequence. The input images are shown in Fig. 3 . The registration result that we expect, having the smoothness of motion in mind, is the alignment of the inner ring with the others, while treating the outer ring as a separate structure. We compare our method to the groupwise registration method proposed by Balci et al. [2]. The results for the two methods are shown in Fig. 3. Looking at the final alignment in Balci's case, it is clear that it does not fulfill the expectations since the outer ring is aligned with the others instead of the inner, real one. In our case, however, the outer ring is identified correctly and and the inner one is aligned with the others. These findings are also supported by the STD and mean images shown in the same figure. The reason for the failure of Balci's method and the success of our method is the consideration of the temporal relationship during the registration, making the method more robust against outliers, as noted in 3.52 . 


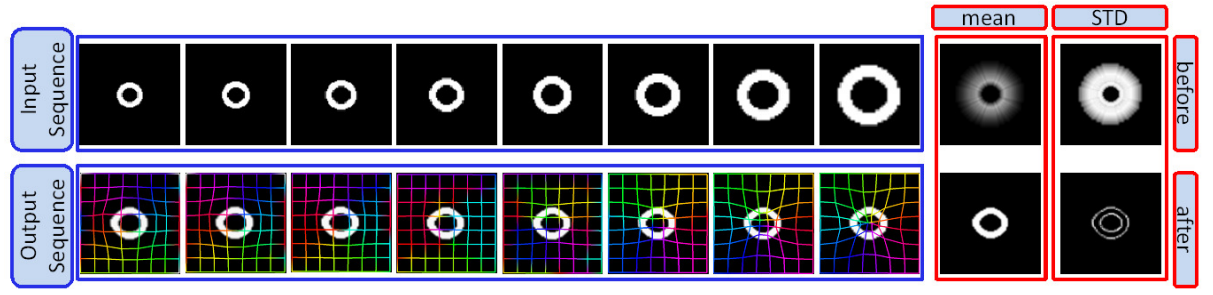

Fig. 1. Input and output sequences together with the statistics images for growing ring experiment

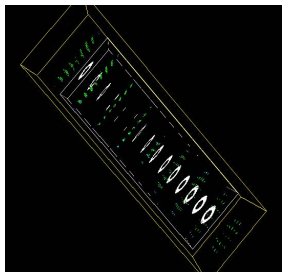

(a)

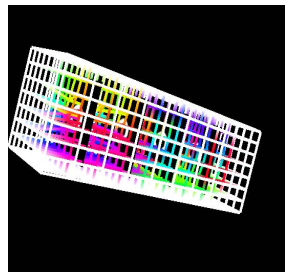

(b)

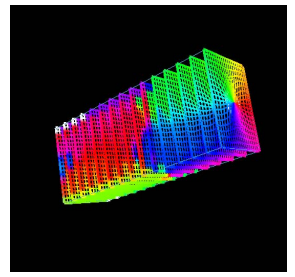

(c)

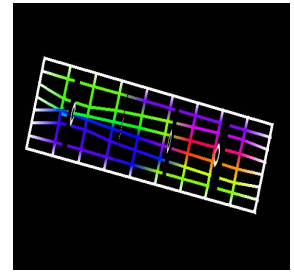

(d)

Fig. 2. (a) $(N+1) \mathrm{D}$ image. (b) $(N+1) \mathrm{D}$ wireframe mesh. (c) $N \mathrm{D}$ meshes in $(N+1) \mathrm{D}$. (d) A cut-plane from the $(N+1) \mathrm{D}$ mesh along the temporal direction.

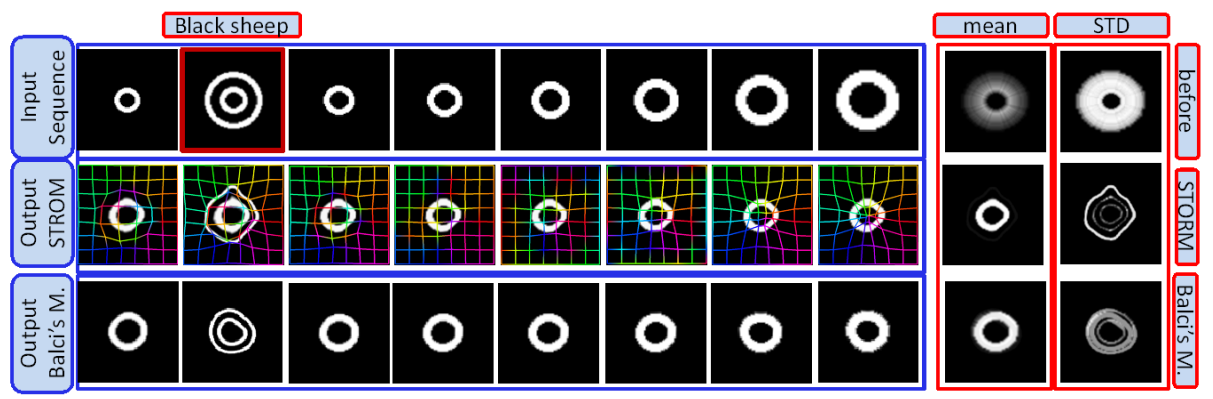

Fig. 3. Input and output sequences together with the statistics images for the black sheep experiment using our method and Balci's method

\section{First vs. Last}

In this case, STORM is tested on a data set with large displacements between the first and last image to model large motion deformations. A randomly created B-spline deformation field is asymmetrically applied to a template image of size $100 \times 100$ pixels. The goal of this experiment is to show the advantage mentioned in 3.53 and the insufficiency of pairwise registration in such cases. In Fig. 4, the input sequence is shown where the middle image is used for creating the sequence. The results in the same figure show that the images are aligned up to some small structures which are difficult for many registration algorithms using FFD's. For the pairwise registration, after trying different implementations of demons algorithm available in ITK, we decided to use the 


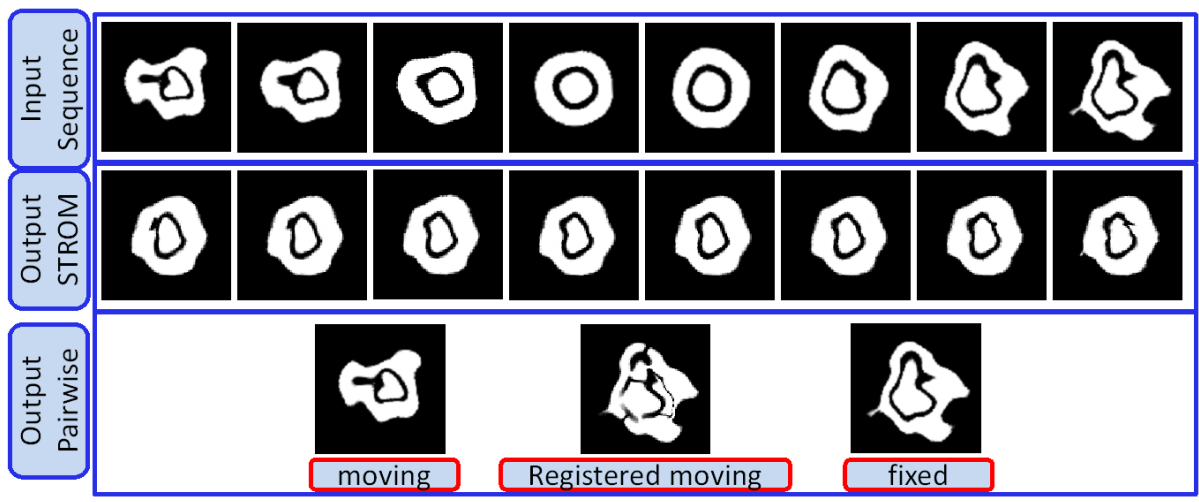

Fig. 4. Input and output sequences together with the statistics images for first vs. last experiment. Last row: Results for experiment with pairwise registration method.

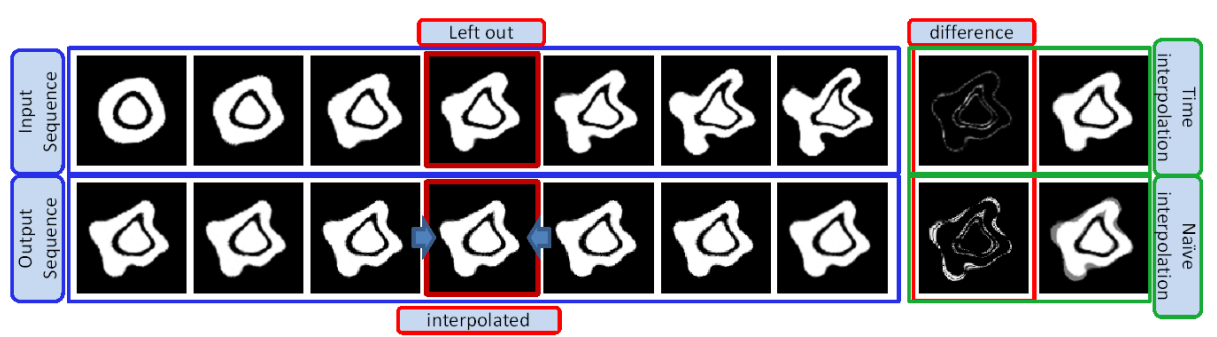

Fig. 5. Illustration of interpolation in time using STORM. Time interpolation and naïve interpolation are compared by showing the difference images to the original image.

diffeomorphic demons proposed by Vercauteren et al. [24], since it achieved the best performance. After registering the first image (moving) to the last image (fixed) in the sequence, we show the deformed moving image in Fig. 4. The dissimilarity between the registration result and the fixed image shows that the large deformation is difficult to handle by pairwise methods.

\section{Time Interpolation}

In this experiment, we want to illustrate the advantage mentioned in 3.5, 4; the interpolation of images along the temporal dimension. For this we use the sequence in Fig. 5, where we omit the indicated image and try to reconstruct it. First, the registration of the reduced sequence is performed. Second, the temporally adjacent images to the reconstructed time point are selected. Third, a linear intensity interpolation between the neighboring, deformed images is performed - with more emphasis to the temporally closer image. Fourth, the inverse transformation is applied to the interpolated image [18], creating the reconstruction. For validation, we compare the reconstructed image to the original image in the sequence by calculating the difference image. Further, we perform a naïve reconstruction by intensity interpolation of the input images. The results clearly show the good performance of the proposed time interpolation. 


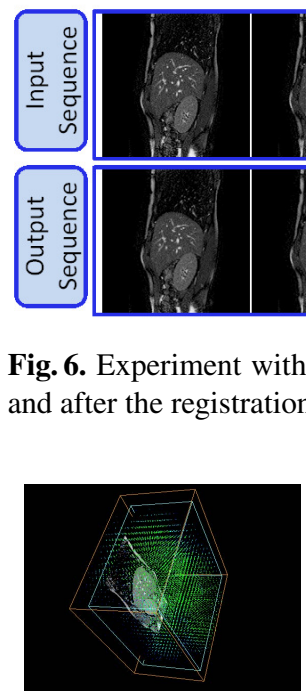

(a)

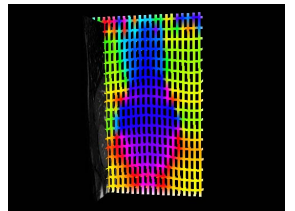

(b)

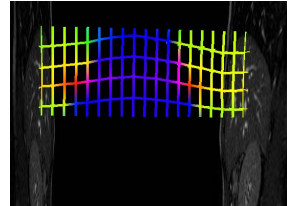

(c)

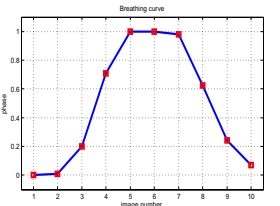

(d)

Fig. 7. (a) $(N+1)$ D deformation field. (b) A temporal slice from $(N+1) \mathrm{D}$ mesh. (c) A closer look into the mesh in (b). (d) The breathing curve for the data set. Note the similarity between the curves in (c) and the curve in (d). The color coded deformation on the mesh shows the direction of motion along the time-axis. Individual lines can be seen as the local motion paths.

\subsection{Medical Data}

Two experiments have been conducted. First, a time-resolved image sequence consisting of $10 \mathrm{MR}$ images is used. The images are so called "navigator slices" that are used for 4D MRI reconstruction in [26]. The images of size 255x255 pixels and temporal resolution $2.7 \mathrm{~Hz}$ are acquired from the liver using the sagittal plane with Philips 1.5T Achieva during one respiratory cycle. The second experiment is done with a CT sequence extracted from the POPI-model [23] which consists of 10 volumes corresponding to phases of a respiratory cycle1. The images are of size $482 \times 141$ pixels and correspond to the sagittal planes in the posterior thoracic region. The same registration settings are used with an additional multi-level grid scheme.

\section{MRI Sequence}

An excerpt from the 10 images in the sequence is shown in Fig. 6. The first and the last images in the sequence correspond to the exhale state of the respiration. We perform registration on the sequence using STORM, with the results being presented in the same figure. The visual inspection and also the calculated statistics images show the good performance of our method for aligning the sequence, and therefore its applicability for registering real medical images. We present the $(N+1)$ D deformation field and

\footnotetext{
${ }^{1}$ The data was obtained from the the Léon Bérard Cancer Center \& CREATIS lab, Lyon, France. http://www.creatis.insa-lyon.fr/rio/popi-model
} 


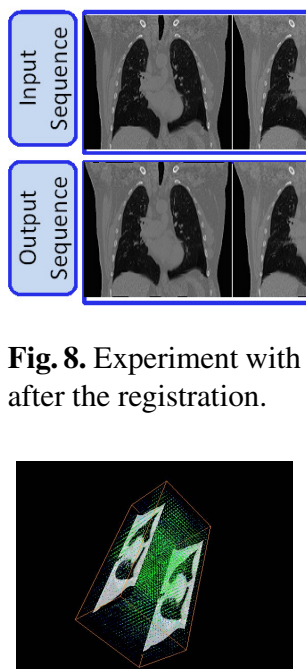

(a) after the registration.

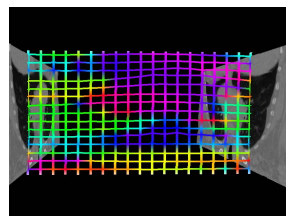

(b)

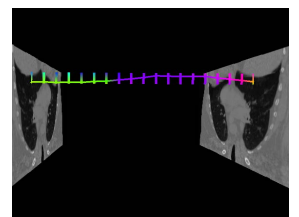

(c)
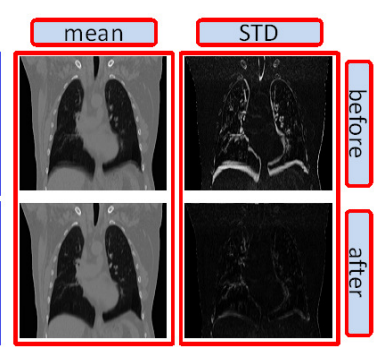
d

Fig. 9. (a) $(N+1)$ D deformation field. (b) A temporal slice from $(N+1)$ D mesh. (c) A closer look into the mesh in (b). (d) The breathing curve for the data set. Note the similarity between the curves in (c) and the curve in (d). The color coded deformation on the mesh shows the direction of motion along the time-axis. Individual lines can be seen as the local motion paths.

a slice along the temporal dimension from the $(N+1)$ D mesh in Figs. 7(a) and 7(b) Furthermore, we recover the breathing curve for this sequence using the image-based gating technique proposed in [28], see Fig. 7(d)] The similarity between the breathing curve and the deformation field in temporal direction in Fig. 7(c) suggests the correspondence between the motion track recovered by both methods.

\section{CT Sequence}

We perform registration using 10 images. The results are presented in Fig. 8 The mean and the STD images in the same figure show the very good performance of STORM on the thoracic images. The high STD around the upper edge of the liver is due to the image construction artifacts, which is quite visible in the input images. We again present images of $(N+1) \mathrm{D}$ deformation field and mesh. Furthermore, as done for the MRI sequence, we recover the breathing curve, see Fig.9(d) The breathing signal from manifold learning is once again similar to the recovered motion trajectories of STORM.

\section{Discussion and Conclusion}

In this work, we have proposed a novel approach to the registration of time-resolved image sequences by taking advantage of groupwise registration. Unlike the traditional methods, a single higher dimensional B-splines based transformation was used. This provides the possibility to have a deformation field also in between the images. Thanks 
to this feature, we were able to interpolate images in the temporal direction, which is necessary in many applications. Recently proposed APE was adapted to the registration of time-resolved images sequences. For optimization, we used stochastic gradient descent optimizer with a line search.

Experiments on synthetic images revealed STORM's advantages in different scenarios over the classical pairwise and groupwise registration methods. It was shown that STORM is more robust against outliers and large deformations within the sequence. The results showed that the use of temporal information ensures the consistency and smoothness in the deformation field along the temporal direction. Moreover, the integration of groupwise registration helped to handle large deformations within the sequence. We performed time interpolation by reconstructing images at any time point in the sequence, which is especially important for applications like radiotherapy treatment planning or 4D image reconstruction. Experiments on CT and MRI sequences showed the good performance of STORM on real medical images.

Acknowledgment. This work was partly funded by the European Commission.

\section{References}

1. Bai, W., Brady, S.: Spatio-temporal image registration for respiratory motion correction in pet imaging. In: ISBI, pp. 426-429. IEEE, Los Alamitos (2009)

2. Balci, S., Golland, P., Wells, W.: Non-rigid Groupwise Registration using B-Spline Deformation Model. In: Workshop on Open-Source and Open-Data for 10th MICCAI, pp. 105-121. Citeseer (2007)

3. Bystrov, D., Vik, T., Schulz, H., Klinder, T., Schmidt, S.: Local motion analysis in 4D lung CT using fast groupwise registration. In: Proceedings of the 16th IEEE International Conference on Image Processing, pp. 1729-1732. IEEE Press, Los Alamitos (2009)

4. Castillo, E., Castillo, R., Martinez, J., Shenoy, M., Guerrero, T.: Four-dimensional deformable image registration using trajectory modeling. PMB 55, 305 (2010)

5. Colgan, R., McClelland, J., McQuaid, D., Evans, P., Hawkes, D., Brock, J., Landau, D., Webb, S.: Planning lung radiotherapy using 4D CT data and a motion model. Physics in Medicine and Biology 53, 5815 (2008)

6. Crum, W., Hartkens, T., Hill, D.: Non-rigid image registration: theory and practice. British Journal of Radiology 77(Special Issue 2), S140 (2004)

7. Ehrhardt, J., Werner, R., Frenzel, T., Säring, D., Lu, W., Low, D., Handels, H.: Reconstruction of $4 \mathrm{D}-\mathrm{CT}$ data sets acquired during free breathing for the analysis of respiratory motion. In: Proc. of SPIE, vol. 6144, pp. 365-372 (2006)

8. Flampouri, S., Jiang, S., Sharp, G., Wolfgang, J., Patel, A., Choi, N.: Estimation of the delivered patient dose in lung IMRT treatment based on deformable registration of 4D-CT data and Monte Carlo simulations. Physics in Medicine and Biology 51, 2763 (2006)

9. Joshi, S., Davis, B., Jomier, M., Gerig, G.: Unbiased diffeomorphic atlas construction for computational anatomy. NeuroImage 23, 151-160 (2004)

10. King, A., Buerger, C., Schaeffter, T.: Cardiac Respiratory Motion Modelling by Simultaneous Registration and Modelling from Dynamic MRI Images. In: WBIR, pp. 222-233 (2010)

11. Klein, G., Huesman, R.: Four-dimensional processing of deformable cardiac PET data. Medical Image Analysis 6(1), 29-46 (2002)

12. Learned-Miller, E.G.: Data driven image models through continuous joint alignment. IEEE Trans on Pattern Analysis and Machine Intelligence 28(2), 236-250 (2006) 
13. Ledesma-Carbayo, M., Kybic, J., Desco, M., Santos, A., Sühling, M., Hunziker, P., Unser, M.: Spatio-temporal nonrigid registration for ultrasound cardiac motion estimation. IEEE Transactions on Medical Imaging 24(9), 1113 (2005)

14. McClelland, J., Blackall, J., Tarte, S., Chandler, A., Hughes, S., Ahmad, S., Landau, D., Hawkes, D.: A continuous 4D motion model from multiple respiratory cycles for use in lung radiotherapy. Medical Physics 33, 3348 (2006)

15. Perperidis, D., Mohiaddin, R., Rueckert, D.: Spatio-temporal free-form registration of cardiac MR image sequences. Medical Image Analysis 9(5), 441-456 (2005)

16. Peyrat, J., Delingette, H., Sermesant, M., Xu, C., Ayache, N.: Registration of 4D Cardiac CT Sequences Under Trajectory Constraints With Multichannel Diffeomorphic Demons. IEEE T-MI (2010)

17. Rohlfing, T., Maurer Jr., C., O’Dell, W., Zhong, J.: Modeling liver motion and deformation during the respiratory cycle using intensity-based nonrigid registration of gated MR images. Medical Physics 31, 427 (2004)

18. Rueckert, D., Aljabar, P., Heckemann, R.A., Hajnal, J.V., Hammers, A.: Diffeomorphic registration using B-splines. In: Larsen, R., Nielsen, M., Sporring, J. (eds.) MICCAI 2006. LNCS, vol. 4191, pp. 702-709. Springer, Heidelberg (2006)

19. Rueckert, D., Sonoda, L., Hayes, C., Hill, D., Leach, M., Hawkes, D.: Nonrigid registration using free-form deformations: application to breast MR images. IEEE Transactions on Medical Imaging 18(8) (1999)

20. Sarrut, D., Boldea, V., Miguet, S., Ginestet, C.: Simulation of four-dimensional CT images from deformable registration between inhale and exhale breath-hold CT scans. Medical Physics 33, 605 (2006)

21. Schreibmann, E., Chen, G., Xing, L.: Image interpolation in 4D CT using a BSpline deformable registration model. IJROBP 64(5), 1537-1550 (2006)

22. Sundar, H., Litt, H., Shen, D.: Estimating myocardial motion by 4D image warping. Pattern Recognition 42(11), 2514-2526 (2009)

23. Vandemeulebroucke, J., Sarrut, D., Clarysse, P.: The popi-model, a point-validated pixelbased breathing thorax model. In: ICCR, Toronto, Canada (2007)

24. Vercauteren, T., Pennec, X., Perchant, A., Ayache, N.: Diffeomorphic demons using ITK's finite difference solver hierarchy. The Insight Journal (2008)

25. Viola, P., Wells III, W.: Alignment by maximization of mutual information. International Journal of Computer Vision 24(2), 137-154 (1997)

26. Von Siebenthal, M., Szekely, G., Gamper, U., Boesiger, P., Lomax, A., Cattin, P.: 4D MR imaging of respiratory organ motion and its variability. PMB 52, 1547 (2007)

27. Wachinger, C., Navab, N.: Similarity Metrics and Efficient Optimization for Simultaneous Registration. In: CVPR (2009)

28. Wachinger, C., Yigitsoy, M., Navab, N.: Manifold learning for image-based breathing gating with application to 4D ultrasound. In: Jiang, T., Navab, N., Pluim, J.P.W., Viergever, M.A. (eds.) MICCAI 2010. LNCS, vol. 6362, pp. 26-33. Springer, Heidelberg (2010)

29. Wu, G., Wang, Q., Jia, H., Shen, D.: Registration of longitudinal image sequences with implicit template and spatial-temporal heuristics. In: Jiang, T., Navab, N., Pluim, J.P.W., Viergever, M.A. (eds.) MICCAI 2010. LNCS, vol. 6362, pp. 618-625. Springer, Heidelberg (2010)

30. Zöllei, L., Learned-Miller, E., Grimson, E., Wells, W.: Efficient population registration of 3D data. In: Computer Vision for Biomedical Image Applications, pp. 291-301 (2005) 